\title{
THE KUIPER BELT
}

\author{
JANE LUU ${ }^{1}$ \\ Physics Department, \\ Stanford University \\ Stanford, CA 94305, USA
}

\begin{abstract}
The existence of a belt of comets in the outer solar system (the "Kuiper belt") has been postulated for a variety of reasons, including the need for a source for the short-period comets. The existence of the belt seems supported by the discoveries of the trans-Neptunian objects $1992 \mathrm{QB}_{1}, 1993 \mathrm{FW}, 1993 \mathrm{RO}, 1993 \mathrm{RP}, 1993 \mathrm{SB}$, and 1993 SC. If these objects are members of the Kuiper belt, crude lower limits on the belt population can be established from the discoveries. The Kuiper belt comets are likely to be primordial remnants of the disk from which the solar system accreted. According to the current theories of cometary nucleus evolution, these objects are expected to possess mantles ("irradiation mantles") which are different from mantles of comets which have been heated to the point of sublimation ("rubble mantles"). Kuiper belt comets on their way to short-period comet orbits may exist among the Centaur objects.
\end{abstract}

\section{Introduction}

Nearly 200 yrs after Edmond Halley discovered that comets were permanent members of the solar system, Jan Oort wrote his classic paper on the origin of comets (Oort 1950). He proposed the existence of the "Oort cloud", a vast swarm of $10^{12}$ comets which lies at the edge of the solar system and forms the source of the long-period (LP) comets (orbital periods $>200 \mathrm{yrs)}$ ). Oort's theory is still accepted today. However, the notion of the Oort cloud also supplying the short-period (SP) comets (orbital periods $<200 \mathrm{yrs}$ ) has come under attack in the last two decades. Joss (1973) pointed out that the Oort cloud could not adequately explain the observed flux of the SP comets. This problem might be solved if a very massive $(\sim$ $700 M_{E a r t h}$, where $M_{E a r t h}$ is one Earth mass) inner Oort cloud existed (Bailey 1986). Alternatively, it was suggested that the SP comets were derived from a belt of low-inclination comets beyond the orbit of Neptune (e.g., Fernández 1980). The latest serious challenge of the Oort cloud as the major source of the SP comets came from Duncan et al. (1988), who demonstrated that the inclination distribution of comets with large perihelia (perihelion distance $q \leq 30 \mathrm{AU}$ ) is conserved during capture. As a consequence, the prograde, low-inclination orbits of the SP comets were incompatible with a spherical source such as the Oort cloud. Duncan et al. then reiterated the hypothesis that a better alternative source may be found in a comet belt beyond the outer planets.

The concept of a population of trans-Neptunian objects is not a new one. It was first championed by Gerard Kuiper, who did not like the apparent emptiness of the outer solar system (Kuiper 1951). Kuiper saw no reason for accretion to end

${ }^{1}$ Hubble Fellow 
abruptly at Neptune. As he saw it, the planetary system formed by coagulation in a disk of gas and dust orbiting the Sun. Near the outer edge of the disk, the icydusty material condensed into comet-size planetesimals which later agglomerated to form the cores of the outer planets. Planet formation stopped at Neptune because, beyond this distance, the low disk density combined with the progressively longer orbital periods to prohibit the formation of planet-size bodies on a reasonable time scale $\left(\sim\right.$ a few $10^{7}$ yrs $)$. However, a disk of icy planetesimals could exist, and lying beyond the outer planets, may be able to survive until today. This disk of partially agglomerated icy planetesimals, christened the Kuiper belt, was presumed to lie in the 40-100 AU region, just beyond Neptune. From the massive cores of Uranus and Neptune (several tens of Earth masses), Kuiper estimated that his disk of icy planetesimals was comparably massive.

Following Kuiper, the concept of a residual comet belt in the outer solar system was investigated by numerous researchers (e.g., Whipple 1964, Cameron 1972, Safronov 1972, Fernández 1980). Fernández and Ip (1981) showed that such a belt could be stable over the age of the solar system, and Duncan et al. (1989) discovered stable bands between Saturn and Uranus and between Uranus and Neptune, which may contain members from the Belt. In this paper I will concentrate on the observational aspects of the Kuiper belt. The interested reader who wishes to learn more about the dynamical aspects of the Kuiper belt is referred to reviews by Fernández and Ip (1991) and Duncan and Quinn (1993).

\section{Observations of the Kuiper Belt}

The Oort cloud is completely unobservable with current technology and thus escapes the scrutiny of observers (a Halley-size comet at distance $10^{4} \mathrm{AU}$ would have magnitude 61). But the Kuiper belt is not so fortunate. At heliocentric distance $R \sim 40 \mathrm{AU}$ (roughly the inner edge of the belt), its largest members are within the reach of available optical telescopes.

The earliest surveys for outer solar system objects employed photographic plates and yielded the planet Pluto (Tombaugh 1961) and the distant comet Chiron (Kowal 1989). The first survey based on modern technology, (i. e., CCDs) was performed by Luu and Jewitt (1988), who covered 0.34 square degrees to the limiting magnitude $m_{R}=24$ mag with the Michigan-Dartmouth-MIT 1.3-m telescope on Kitt Peak, Arizona. Their strategy was as follows :

1) Consecutive images were taken of fields centered on the ecliptic. The ecliptic plane (or near it) is where most solar system objects, including the SP comets, are likely to be concentrated.

2) The fields have to be located near opposition, in order to allow the estimation of the distance of any object found during the survey. At opposition, most of an object's motion is due to parallax. Assuming concentric, coplanar circles for the orbits of the Earth and the Kuiper belt candidate, the apparent motion, $d \theta / d t$ $[\operatorname{arcsec} / \mathrm{hr}]$ of the candidate is

$$
\frac{d \theta}{d t}=148\left(\frac{1--R^{-0.5}}{R--1}\right)
$$


where $R>1$ is the heliocentric distance in AU.

3) Observations for the survey were carried out at the time of the equinoxes, when the Milky Way is at high ecliptic latitude. This minimizes the density of field stars and facilitates the identification of faint, slow moving objects.

4) Three consecutive images were taken of each field, followed by a fourth image separated by at least $2 \mathrm{hrs}$ in time. The images were then displayed rapidly ("blinked") to detect moving objects. Although 2 images are sufficient to identify motion, the redundancy eliminates confusion with cosmic ray hits in the field.

More detail on the search strategy can be found in Luu and Jewitt (1988). The survey found no new objects beyond Saturn.

The Luu and Jewitt survey was followed by searches by several other groups, the largest of which was carried out by Levison and Duncan (1990), who used the U. S. Naval Observatory 40-in. telescope to cover nearly 5 sq. degrees to $m_{V}=$ $22.5\left(m_{R} \sim 22\right)$. No evidence for the Kuiper belt was found until August 1992, when Jewitt and Luu (1993a) discovered the faint, slow-moving object $1992 \mathrm{QB}_{1}$ with the University of Hawaii $2.2 \mathrm{~m}$. Other discoveries followed quickly : the second Kuiper belt candidate, $1993 \mathrm{FW}$, was found 6 months later, followed by 4 more objects (1993 RO, 1993 RP, 1993 SB, 1993 SC) 6 months after that. The discoveries are summarized in Table I.

TABLE I

Trans-Neptunian Objects

\begin{tabular}{|c|c|c|c|c|c|c|}
\hline Object & $\begin{array}{c}a \\
{[\mathrm{AU}]}\end{array}$ & $e$ & $\begin{array}{l}i \\
{[\mathrm{deg}]}\end{array}$ & Mag & $\begin{array}{c}\text { Discovery } \\
\text { Date }\end{array}$ & Discoverers \\
\hline $1992 \mathrm{QB}_{1}$ & 43.8 & 0.07 & 2.20 & 22.8 & Aug 1992 & Jewitt and Luu (1993a) \\
\hline $1993 \mathrm{FW}$ & 42.4 & 0.04 & 7.75 & 22.8 & Mar 1993 & Luu and Jewitt (1993a) \\
\hline $1993 \mathrm{RO}$ & 32.3 & $0.00^{a}$ & 2.53 & 23.2 & Sep 1993 & Jewitt and Luu (1993b) \\
\hline 1993 RP & 35.4 & $0.00^{a}$ & 2.80 & 24.5 & Sep 1993 & Luu and Jewitt (1993b) \\
\hline $1993 \mathrm{SB}$ & 33.2 & $0.00^{a}$ & 2.28 & 22.7 & Sep 1993 & Williams et al. (1993) \\
\hline 1993 SC & 34.5 & $0.00^{a}$ & 5.57 & 21.7 & Sep 1993 & Williams et al. (1993) \\
\hline
\end{tabular}

The problem of determining the orbits of the newly discovered trans-Neptunian objects is much more difficult than that involving asteroids and comets in the inner planetary region, due to the large distances and long orbital periods of the transNeptunian objects. Continuing astrometry is essential, yet the task is complicated 
by these objects' faint appearances. $1992 \mathrm{QB}_{1}$ has been confirmed to have a nearly circular orbit beyond Neptune (Marsden 1993) but it is yet too early to tell for the other objects.

A graphical summary of recent outer solar system surveys can be found in Fig. 1 , which plots the surface densities of the searches versus their limiting magnitudes. The Figure seems to suggest that flux-limited surveys (i.e., all the mentioned searches) are likely to succeed by covering at least $\sim 1$ sq. deg. to $m_{R} \sim 23$.

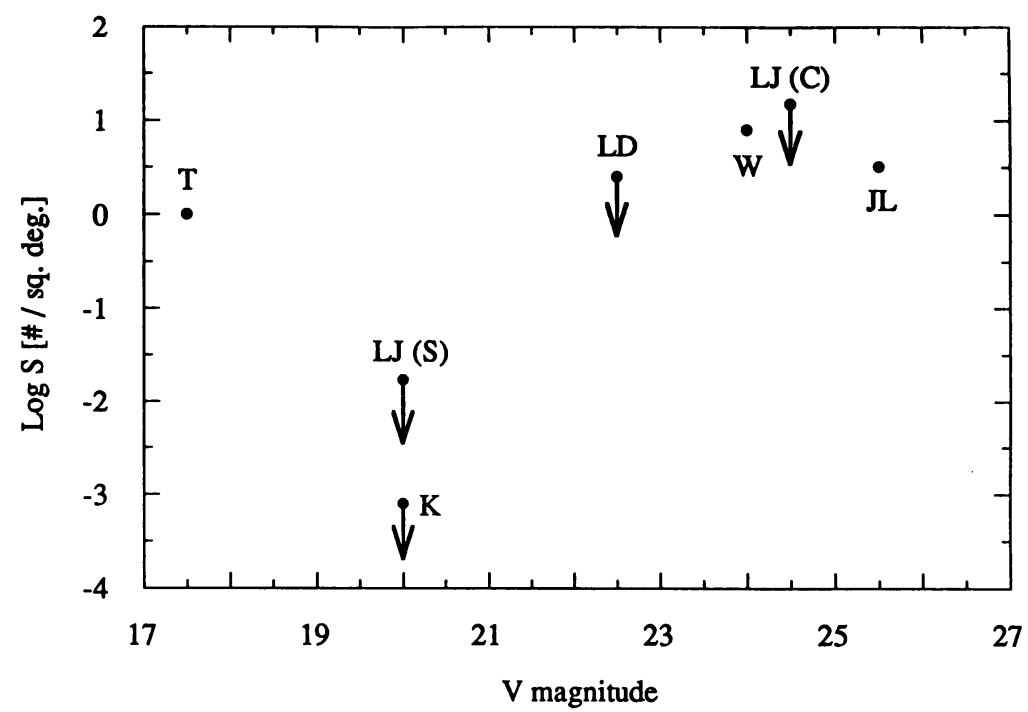

Fig. 1. Surface density of objects beyond Neptune as a function of limiting magnitudes for various surveys. Downward arrows indicate $99 \%$-confidence upper limits. The symbols are : $\mathrm{T}=$ Tombaugh (1961), $\mathrm{K}=$ Kowal (1989), LJ $(\mathrm{S})=$ Luu and Jewitt (1988, with Schmidt ), LJ (C) = Luu and Jewitt (1988, with CCD), LD = Levison and Duncan 1990, $\mathrm{JL}=$ Jewitt and Luu (1993), $\mathrm{W}=$ Williams et al. (1993).

\section{The Kuiper Belt}

A sample of six objects hardly allows meaningful statistical analysis, and furthermore, it is not clear that all six objects belong to the postulated Kuiper belt. In this section, for the sake of argument, we assume that they are members of the belt, and we explore the implications of the discoveries upon the Kuiper belt population.

\section{1. MASS AND POPULATION}

The six objects were detected in $\sim 1.5$ sq. degree of the sky. $1993 \mathrm{FW}$ currently possesses the most inclined orbit at $\sim 8 \mathrm{deg}$. The projected area of the belt as seen from the Earth is thus $2 \times 8 \mathrm{deg} \times 360 \mathrm{deg}$, or $5760 \mathrm{sq}$. degrees. The extrapolated minimum number of objects brighter than $m_{R} \sim 25$ is then $N_{\min } \sim 2 \times 10^{4}$ objects. 
Assuming a density of $1000 \mathrm{~kg} \mathrm{~m} \mathrm{~m}^{-3}$, the minimum mass of the belt (counting only objects with diameter larger than $\sim 100-\mathrm{km})$ is $M \geq 0.03 M_{\text {Earth }}$.

It should be emphasized that this estimated mass can be no more than a lower limit, as all the surveys up to date are necessarily biased toward the largest, closest members of the belt. Smaller, as well as more distant, objects must exist which are currently beyond the practical limit of ground-based telescopes. Furthermore, the Kuiper belt is likely to be thicker than $16 \mathrm{deg}$ (the inclination distribution of the SP comets is $\sim 30 \mathrm{deg}$ ). We note that this minimum mass is consistent with previous mass estimates of the Kuiper belt. The absence of perturbations on the orbit of comet Halley placed an upper limit of $M_{\text {belt }} \leq 1 M_{E a r t h}$ (Hamid et al. 1968, Yeomans 1986, Hogg et al. 1991), while Duncan et al. (1988) estimated at least $M_{\text {belt }} \sim 0.02 M_{\text {Earth }}$ was needed to produce the observed flux of SP comets. Stronger constraints on the mass of the Kuiper belt can only be reached with an understanding of the size and spatial distribution of the belt.

We know very little about the size distribution of the Kuiper belt. If it resembles the asteroid belt, the familiar asteroidal power-law size distribution predicts that large objects should be more abundant than smaller objects. So far, the surveys have yielded fewer faint objects near the detection limit $\left(m_{R} \sim 25\right)$ than the relatively bright objects such as $1992 \mathrm{QB}_{1}$. The apparent "scarcity" of smaller objects may be understood if we keep in mind that the Jewitt and Luu survey is flux-limited, not volume-limited. In a flux-limited survey, the limiting magnitude determines the volume of space, as well as the size range, that the survey can sample. Because of the dependence on geometry, the apparent size distribution is most likely not the true size distribution. Furthermore, the Kuiper belt size distribution may also be very different from an asteroidal size distribution. Again, the character of the belt population can only be revealed with a larger sample of possible belt members.

\section{2. DYNAMICS}

The dynamical properties of the belt have not been clearly established. Although the belt has been identified as an alternative source for SP comets, dynamical processes operating within the belt are poorly understood. The various mechanisms suggested to transfer comets into the inner solar system include perturbations by the largest members of the belt (Duncan et al. 1988, Ip and Fernández 1991), and chaos. Torbett and Smoluchowski (1990) discovered a chaotic zone beyond Neptune for objects with perihelia between 30 and 45 AU. Holman and Wisdom (1993) confirmed the important role of chaos in delivering SP comets from the Kuiper belt, and pointed out that the delivery process may be analogous to the delivery of meteorites from resonances in the asteroid belt (Wisdom 1985, Froeschlé and Scholl 1986). Numerical simulations also revealed that the belt was likely to have an inner edge near $40 \mathrm{AU}$ (Holman and Wisdom 1993), beyond which existed stable regions interspersed with unstable regions. Although its inner edge may be slowly eroded, the Kuiper belt thus could survive for the age of the solar system and can be the source of the SP comets (Gladman and Duncan 1990, Levison 1991).

The orbit of $1992 \mathrm{QB}_{1}$ appears stable against perturbations by Neptune on timescales of $10^{9}$ yrs (Levison and Duncan 1993). At the present time, 1993 RO, 
$1993 \mathrm{RP}, 1993 \mathrm{SB}$ and $1993 \mathrm{SC}$ have semimajor axes estimated at 32-35 AU. If these estimates are correct, some of these objects are likely to have future close encounters with Neptune. Once on Neptune-crossing orbits, the comets may be on their way to the inner planetary region. A fraction of the Neptune-crossing comets will be gravitationally scattered by the giant planets into Jupiter-crossing orbits, from which they may become observable SP comets (Everhart 1977). The timescale in which the transfer process takes place is short compared to the age of the solar system $\left(10^{5}--10^{7} \mathrm{yrs}\right.$, Gladman and Duncan 1990). Is it then possible to observe Kuiper belt comets during their journey to the inner planetary region?

We may witness some of these transition comets in the Centaur population. Centaurs are objects on elliptical orbits which cross the orbits of one or more giant planets. Planet-crossing orbits are very unstable, evolving on the timescale of $10^{5}--10^{6}$ yrs (Oikawa and Everhart 1979), implying that the Centaur must have originated somewhere else, quite possibly the Kuiper belt. The known Centaurs currently include 2060 Chiron, 5145 Pholus and $1993 \mathrm{HA}_{2}$, all with semimajor axes in the Saturn-Uranus region. These objects also possess unusual physical properties that suggest a non-asteroidal origin, and they will be discussed in more detail in section 5.2 .

\section{3. FUTURE WORK}

Several surveys of the outer solar system are still in progress, fueled by the evidence that the Kuiper belt may exist and is within the grasp of ground-based telescopes. Most surveys employ medium to large-size telescopes $(\sim 2-4 \mathrm{~m}$ range) and are aimed toward optical detection of the belt comets via reflected sunlight. An alternative observational strategy with great promise relies on the detection of Kuiper belt comets via their occultation of background stars (Bailey 1976, Dyson 1992). Axelrod et al. (1992) outline a survey which would make use of a row of small telescopes equipped with wide field CCDs. The telescopes would monitor a large number of stars of small angular diameter to search for very short duration occultations, during which the stars would appear to blink out because a comet blocks their light. This strategy has the advantage of being more sensitive to more numerous smaller (as opposed to the largest and rarest) Kuiper belt objects, as well as relying only on dedicated small, inexpensive telescopes and is thus impervious to the ubiquitous problem of oversubscription on medium-large telescopes.

\section{Surface Composition : the Irradiation Mantle}

Whether in the Oort cloud or the Kuiper belt, comets are continually exposed to galactic cosmic rays, consisting of $\sim 93 \%$ protons, $6 \%$ helium nuclei, and $1 \%$ heavier nuclei (Meyer et al. 1974). The mean energy deposited in a cometary nucleus over 4.5 billion years was independently estimated by Donn (1976) and Whipple (1977) to be $\sim 3 \times 10^{19} \mathrm{MeV} \mathrm{cm}{ }^{-2}$, enough to drive significant chemical modification of the surface layers. For example, irradiation of mixtures of astrophysically relevant ices such as $\mathrm{H}_{2} \mathrm{O}, \mathrm{NH}_{3}, \mathrm{CH}_{4}, \mathrm{~N}_{2}, \mathrm{CO}$ and $\mathrm{CO}_{2}$ with a dose of $\sim 100$ $\mathrm{eV} /$ molecule produced $\mathrm{C}_{2} \mathrm{H}_{6}, \mathrm{CO}_{2}, \mathrm{CO}, \mathrm{N}_{2} \mathrm{O}$, NO and $\mathrm{CH}_{4}$ (see TABLE II, also 
review by Strazzulla and Johnson 1991). The typical dose estimated to be deposited in the top 20-cm layer of a comet stored in the Oort cloud is $\sim 40 \mathrm{eV} /$ molecule (Moore et al. 1983). It is not clear what fraction of the newly formed radicals and molecules will survive until the comet's first entry into the planetary region. What is postulated to survive the residence time in the Oort cloud/Kuiper belt, however, is a crust of nonvolatile organic residue (Moore et al. 1983, Johnson et al. 1987, Khare et al. 1989). This residue has been observed in laboratory experiments with irradiated ices, and is formed by bombardment of carbon and sulfur-containing species. Evidence for such organic refractory residues in space exists in the $3.4 \mu \mathrm{m}$ feature seen in the galactic center sources IRS7 and Sgr AW, and identified with residues created in the laboratory (Greenberg 1987). The formation of the residue appears independent of the particle energy in the range $\sim 100 \mathrm{eV}$ to $1 \mathrm{MeV}$.

TABLE II

Identified products of irradiated ices ${ }^{a}$

\begin{tabular}{lrr}
\hline \hline Irradiated Ices & Irradiation type & Identified products \\
\hline $\mathrm{H}_{2} \mathrm{O}+\mathrm{NH}_{3}+\mathrm{CH}_{4}$ & proton & $\mathrm{C}_{2} \mathrm{H}_{6}, \mathrm{CO}_{2},{ }^{13} \mathrm{CO}_{2}$, \\
$\mathrm{H}_{2} \mathrm{O}+\mathrm{N}_{2}+\mathrm{CO}_{2}$ & proton & $\mathrm{CO}, \mathrm{N}-\mathrm{N}-\mathrm{N}$ species \\
$\mathrm{H}_{2} \mathrm{O}+\mathrm{N}_{2}+\mathrm{CO}$ & & ${ }^{12} \mathrm{CO},{ }^{13} \mathrm{CO}, \mathrm{NO}$, \\
& proton & ${ }^{12} \mathrm{CO}_{2},{ }^{13} \mathrm{CO}_{2}, \mathrm{CO}_{2}$, \\
$\mathrm{NH}_{3}+\mathrm{CO}_{2}$ & $\mathrm{UV}$ & $\mathrm{CN}, \mathrm{HCO}, \mathrm{NH}_{2}, \mathrm{NH}_{2} \mathrm{CO}_{2}$ \\
& & $\mathrm{CO}_{2}, \mathrm{H}_{2} \mathrm{CO}, \mathrm{NH}_{4}^{+}$ \\
$\mathrm{H}_{2} \mathrm{O}+\mathrm{NH}_{3}+\mathrm{CO}+\mathrm{CO}_{2}$ & $\mathrm{UV}$ & $\mathrm{NO}^{+} \mathrm{HCO}_{1} \mathrm{CO}_{2}, \mathrm{NO}_{2}^{-}$, \\
& & $\mathrm{NH}_{4}^{+}, \mathrm{OCN}^{-}, \mathrm{HCONH}_{2}$ \\
\hline \hline
\end{tabular}

a The products of proton-irradiated ices were adopted from Moore et al. (1983), while those of UV-irradiated ices were adopted from Grim et al. (1989).

vertically

The typical chemical reaction when ices are irradiated is the preferential liberation of hydrogen, encouraging the formation of carbon bonds such as $\mathrm{C}-\mathrm{C}, \mathrm{C}-\mathrm{N}$, $\mathrm{C}-\mathrm{O}, \mathrm{C}-\mathrm{S}$. The irradiated ices become increasingly darker as part of the volatile ice mass is converted into the refractory residue (Moore et al. 1983, Khare et al. 1989). The residue thickness is a function of the initial porosity and particle fluence (Strazzulla and Johnson 1991). In general, it is observed to increase proportionally with the received irradiation, and is predicted to reach a few meters over the age of the solar system. The crust of thickened residue is usually referred to as the "irradiation mantle," to be distinguished from the "rubble mantle" which is a direct consequence of sublimation (e.g., Jewitt 1992). The irradiation mantle should cover most of the surface due to the isotropy of the galactic cosmic ray field and the rotation of the nucleus. The irradiated crust is also likely to be porous, with cracks and fissures in the mantle which allow gas diffusion upon heating. The thickness of 
the crust, the gas diffusion capability, together with the slow rate of heating by a comet as it drifts toward the Sun, have led Strazzulla et al. (1991) to believe that a significant part of the crust should be able to survive the first few orbits in the planetary region. A rough illustration of the effects of irradiation upon the nucleus surface is given in Fig. 2.

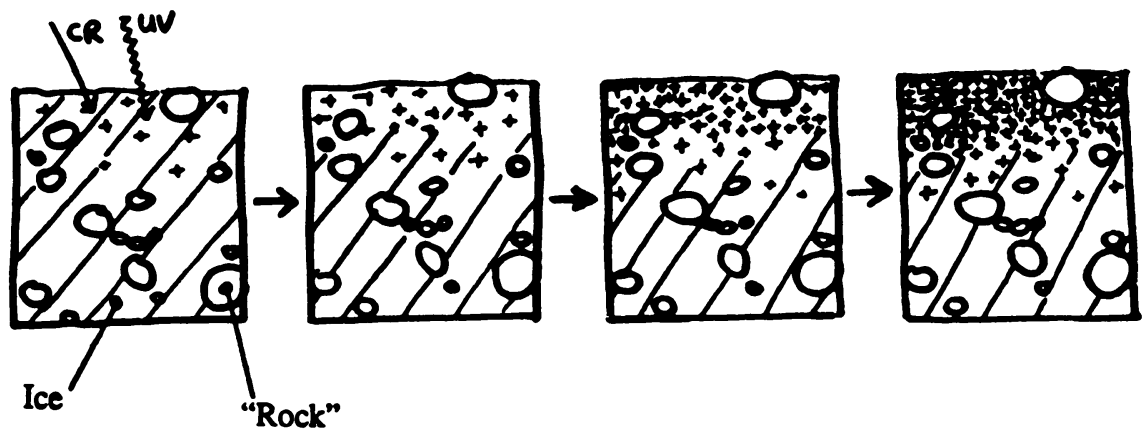

Fig. 2. The creation of an irradiation mantle. The nucleus surface, consisting of ice and rocky material, is irradiated by high energy particles and produces an irradiation-induced crust. Figure adapted from Jewitt : 1992,"

It is not known how or whether comets in the Oort cloud physically differ from those in the Kuiper belt. Both suffer from long-term exposure to cosmic ray irradiation, but where their evolutionary paths diverge is not known. Available observations indicate that the interplanetary cosmic ray intensity should reach the average large scale galactic value at roughly the distance of the belt ( $R \sim 50-60 \mathrm{AU}$, Webber 1987) so the irradiation damage may not be much different between the Oort cloud and Kuiper belt comets. On the other hand, the Kuiper belt straddles the termination shock $(R \sim 50 \mathrm{AU}$, Suess 1990) and the heliopause $(R \sim 80 \mathrm{AU}$, Webber 1987) and may experience additional processing particular to these areas (e.g., cosmic ray production from the interaction of interstellar neutral gas with the solar wind, Suess 1990). It has also been suggested that the Oort cloud comets are heated by passing stars (Stern and Shull 1988), whereas the Kuiper belt comets would be much less susceptible to their effects to their smaller distances. The main effect of UV photons on cometary ices such as $\mathrm{H}_{2} \mathrm{O}, \mathrm{CH}_{4}$ and $\mathrm{NH}_{3}$ is to break the molecular bonds, leaving behind radicals (e.g., TABLE II, also Greenberg 1983). These radicals may remain as is or recombine to form new molecules. Thus, if the heating effect of passing stars is significant, it may result in somewhat different surface compositions between the Oort cloud and Kuiper belt comets. These hypotheses remain untested until observations of the nuclei of long-period comets are available. 


\section{Properties of Kuiper Belt Candidates}

\section{1. THE TRANS-NEPTUNIAN OBJECTS}

At the time of discovery, the apparent magnitudes of the trans-Neptunian objects range from $m_{R} \sim 22.8$ to 24.5. What little is known about their physical properties is summarized in TABLE III. Assuming an albedo of 0.04 (typical of known comet nuclei), they have diameters in the range $100-250 \mathrm{~km}$. The few colors available indicate that they are red, although possibly with different degrees of redness. $1992 \mathrm{QB}_{1}$ and $1993 \mathrm{RO}$ seem to be substantially redder than the Sun, while 1992 FW possesses a more moderate red color.

TABLE III

Properties of Trans-Neptunian Objects

\begin{tabular}{|c|c|c|c|c|}
\hline & $\begin{array}{c}\text { Diameter } \\
{[\mathbf{k m}]}\end{array}$ & $\mathbf{V}-\mathbf{R}$ & R - I & $\begin{array}{l}\text { Cometary } \\
\text { Activity? }\end{array}$ \\
\hline $1992 \mathrm{QB}_{1}$ & $250^{a}$ & $0.6 \pm 0.1^{b}$ & $1.0 \pm 0.2^{b}$ & No \\
\hline $1993 \mathrm{FW}$ & $250^{a}$ & $0.4 \pm 0.1^{c}$ & --- & No \\
\hline 1993 RO & $120^{a}$ & -- & -- & No \\
\hline 1993 RP & $100^{a}$ & $-\cdots$ & -- & No \\
\hline $1993 \mathrm{SB}$ & $160^{a}$ & --- & -- & No \\
\hline $1993 \mathrm{SC}$ & $280^{a}$ & -- & -- & No \\
\hline Solar colors ${ }^{d}$ & & 0.32 & 0.4 & \\
\hline
\end{tabular}

$a_{\text {approximate, assuming an albedo of } 0.04}$

$b$ Jewitt and Luu (1993a)

${ }^{c}$ Luu and Jewitt (1993a)

${ }^{d}$ Fernie (1983) and Hartmann et al. (1990)

We have few constraints on the origin of these objects, since the size distribution is not known. The collision time between bodies with diameters $\geq 100 \mathrm{~km}$ is comparable to or greater than the age of the solar system, but collisions with smaller, more abundant objects are likely. However, collisions in the Kuiper belt occur at lower velocities than in the asteroid belt ( $\mathrm{a}$ few $\mathrm{x} 0.1 \mathrm{~km} \mathrm{~s}^{-1}$ as opposed to a few $\mathrm{x} \mathrm{km} \mathrm{s} s^{-1}$ ) and therefore should be less disruptive. 


\section{2. THE CENTAURS}

A summary of the known physical properties of the Centaurs is provided in TABLE IV.

TABLE IV

Properties of Centaur Objects

\begin{tabular}{|c|c|c|c|c|c|}
\hline & $\begin{array}{l}\text { Diameter } \\
{[\mathrm{km}]}\end{array}$ & $\mathbf{V}-\mathbf{R}$ & R - I & $\underset{\left[\% / 10^{3} \AA\right]}{S^{a}}$ & $\begin{array}{l}\text { Cometary } \\
\text { Activity? }\end{array}$ \\
\hline 2060 Chiron & $\leq 300^{b}$ & $0.34 \pm 0.03^{c}$ & $0.28 \pm 0.03^{c}$ & $-3.9 \pm 0.4^{d}$ & Yes \\
\hline 5145 Pholus & $189 \pm 26^{e}$ & $0.7^{f}$ & $0.72 \pm 0.06^{f}$ & $46.1 \pm 0.3^{d}$ & No \\
\hline $1993 \mathrm{HA}_{2}$ & $60^{g}$ & $\sim 0.7^{h}$ & ---- & ---- & No \\
\hline Solar colors ${ }^{i}$ & & & 0.32 & 0.4 & \\
\hline
\end{tabular}

${ }^{a}$ Normalized reflectivity gradient

$b_{3 \sigma}$ upper limit (Jewitt and Luu 1992)

${ }^{c}$ Hartmann et al. (1990)

$d_{\text {Luu (1993) }}$

$e_{\text {minimum diameter (Davies et al. 1993) }}$

$f_{\text {Mueller et al. (1992) }}$

8 assuming an albedo of 0.04

$h_{\text {Jewitt and Luu 1994, unpublished data }}$

${ }^{i}$ Fernie (1983) and Hartmann et al. (1990)

Classified as an asteroid upon discovery, Chiron is now known to be a comet (Tholen et al. 1988, Meech and Belton 1989). No sign of cometary activity has been detected in Pholus or $1993 \mathrm{HA}_{2}$, but Pholus possesses a very red color (V$\mathrm{R}=0.7$; e.g., Mueller et al. 1992) which may be rivaled only by $1993 \mathrm{HA}_{2}$ and the trans-Neptunian objects (see TABLE III). A good (but non-unique) match for the spectra of Pholus has been found in tholins, organic residues formed by irradiation of $\mathrm{CH}_{4}$ and other organic molecules (Davies and Sykes 1992, Wilson et al. 1994). A tholin-rich surface is consistent with an irradiation mantle and an origin in the Kuiper belt. From thermal measurements, Davies et al. (1993) reported that the albedo of Pholus could not exceed $0.044 \pm 0.013$, corresponding to a minimum diameter of $189 \pm 26 \mathrm{~km}$. The low albedo is similar to that of known cometary nuclei (Jewitt 1991). Recent work by Yabushita (1993) lends emphasis to the irradiation mantle hypothesis by showing that large (radius $\geq 40 \mathrm{~km}$ ) nuclei are especially susceptible to irradiated ices, since they have a layered structure where highly volatile materials are pushed toward the surface. 
The red color of Pholus stands in sharp contrast with the neutral-blue color of Chiron (Luu and Jewitt 1990, Luu 1993). The color difference has been attributed to the presence of cometary activity and the lack thereof on Pholus. In this scenario, sublimation has blown off or covered with debris the original irradiation mantle of Chiron, while that of Pholus remains intact. We caution that this theory is only speculative and can be tested if cometary activity is detected in Pholus or 1993 $\mathrm{HA}_{2}$.

\section{Conclusions}

Our understanding of the putative Kuiper belt and its members may be summarized as follows :

1) The existence of the Kuiper belt is supported by the discovery of the transNeptunian objects 1992 QB $_{1}, 1993$ FW, 1993 RO, 1993 RP, 1993 SB and 1993 SC. The orbit of $1992 \mathrm{QB}_{1}$ is consistent with a residence in the Kuiper belt. The orbits of the other trans-Neptunian objects are still indeterminate at the time of writing. 2) The Kuiper belt is accessible to ground-based studies and, as a fossil from the formation of the solar system, is an important reservoir of primordial material. This reservoir awaits exploration and may yield important information on early solar system conditions.

3) The dynamics of the Kuiper belt remain largely unexplored. Recent numerical simulations suggest that chaos and gravitational scattering by large belt members play a role in the delivery of comets from the belt to the inner planetary region.

4) Kuiper belt objects are expected to retain primordial irradiation mantles, produced by prolonged irradiation of ices by cosmic rays. The irradiation mantles may exhibit diverse colors.

5) The Centaur objects (2060 Chiron, 5145 Pholus, and $1993 \mathrm{HA}_{2}$ ) may be Kuiper belt comets in transition to short-period comet orbits.

\section{Acknowledgements}

I thank MDM Observatories and the University of Hawaii for allocation of telescope time, and Brian Marsden for his invaluable help with orbit determination. Support for this work was provided by NASA through grant \# HF-1035.01-92A awarded by the Space Telescope Science Institute which is operated by the Association of Universities for Research in Astronomy, Inc., for NASA under contract NAS526555.

\section{References}

Axelrod, T. S., Alcock, C., Cook, K. H. and Park, H.-S. : 1992, "A direct census of the Oort Cloud with a robotic telescope." In Robotic Telescopes in the 1990's, (A. V. Filippenko, Ed.), ASP Conference Series, vol. 34, 171-181, Astronomical Society of the Pacific, San Francisco.

Bailey, M. : 1976, "Can "invisible" bodies be observed in the Solar System?" Nature, 259 , 290-291. 
Bailey, M. : 1986, "The mean energy transfer rate to comets in the Oort cloud and implications for cometary origins." Mon. Not. Roy. Astron. Soc., 218, 1-30.

Cameron, A. G. W. : 1972, "The formation of the sun and planets." Icarus, 1, 13-69.

Davies, J. and Sykes, M. : 1992, "IAU Circular 5480".

Davies, J., Spencer, J., Sykes, M., Tholen, D. and Green, S. : 1993, "IAU Circular 5698."

Donn, B. : 1976, "The nucleus : panel discussion." In The Study of Comets (B. Donn et al., Eds.), 611-621, NASA SP-393, Government Printing Office, Washington, DC.

Duncan, M., Quinn, T. and Tremaine, S. : 1988, "The origin of short-period comets." Astrophys. J. Lett., 328, L69-L73.

Duncan, M., Quinn, T. and Tremaine, S. : 1989, "The long-term evolution of orbits in the solar system : a mapping approach." Icarus, 82, 402-418.

Duncan, M. J. and Quinn, T. : 1993, "The long-term dynamical evolution and stability of the solar system." In Protostars and Planets III (E.H. Levy, J.I. Lunine, M.S. Matthews, Eds.), 1371-1394, University of Arizona Press, Tucson.

Dyson, F. J. : 1992, "Hunting for comets and planets." Quart. J. Roy. Astron. Soc., 33, 45-57.

Everhart, E. : 1977, "The evolution of comet orbits as perturbed by Uranus and Neptune." In Comets-Asteroids-Meteorites (A. H. Delsemme, Ed.), 99-107, University of Toledo Press, Ohio.

Fernández, J. A. : 1980, "On the existence of a comet belt beyond Neptune." Mon. Not. Roy. Astron. Soc., 192, 481-491.

Fernández, J. A. and Ip, W. H. : 1981, "Dynamical evolution of a cometary swarm in the outer planetary region." Icarus, 47, 470-479.

Fernández, J. A. and Ip, W. H. : 1991, "Statistical and evolutionary aspects of cometary orbits." In Comets in the Post-Halley Era (R. L. Newburn, Jr., M. Neugebauer, J. Rahe, Eds.), 487-535, Kluwer, Dordrecht.

Fernie, J. D. : 1983, "Relationships between the Johnson and Kron-Cousins VRI photometric systems." P. A. S. P., 95, 782-785.

Froeschlé, Ch. and Scholl, H. : 1986, "The secular resonance $\nu_{6}$ in the asteroidal belt." Astron. Astrophys., 166, 326-336.

Gladman, B., and Duncan, M. : 1990, "On the fate of minor bodies in the outer solar system." Astron. J., 100, 1680-1693.

Greenberg, J.M. : 1983, "The largest molecules in space : interstellar dust." In Cosmochemistry and the Origin of Life (C. Ponnamperuma, Ed.), 71-1, Reidel, Dordrecht.

Greenberg, J. M. : 1987, "Basic laboratory studies of grains." In Astrochemistry (M. S. Vardya, S. P. Tarafdar, Eds.), 501-523, Reidel, Dordrecht.

Grim, R. J. A., Greenberg, J. M., de Greot, M. S., Baas, F., Schutte, W. A. and Schmitt, B. : 1989, "Infrared spectroscopy of astrophysical ices : new insight in the photochemistry." Astron. Astrophys. Suppl. Ser., 78, 161-186.

Hamid, S. E., Marsden, B. and Whipple, F. : 1968, "Influence of a comet belt beyond Neptune on the motions of periodic comets." Astron. J., 73, 727.

Hartmann, W. K., Tholen, D. J., Meech, K. and Cruikshank, D. P. : 1990, "2060 Chiron : Colorimetry and cometary behavior." Icarus 83, 1-15.

Hogg, D. W., Quinlan, G. D. and Tremaine, S. : 1991, "Dynamical limits on dark mass in the outer solar system." Astron. J., 101, 2274-2286.

Holman, M. and Wisdom, J. : 1993, "Dynamical stability in the outer solar system and the delivery of short-period comets." Astron. J., 105, 1987-1999.

Ip, W.-H. and Fernández, J. A. : 1991, "Steady-state injection of short- period comets from the trans-Neptunian cometary belt." Icarus, 92, 185-193.

Jewitt, D. C. : 1991, "Cometary Photometry." In Comets in the Post-Halley Era (R. L. Newburn, Jr., M. Neugebauer, J. Rahe, Eds.), 19-66, Kluwer, Dordrecht.

Jewitt, D. C. : 1992, "Physical Properties of Cometary Nuclei." In Proceedings of the 30th Liege International Astrophysical Colloquium (A. Brahic, J.-C. Gerard, J. Surdej, Eds.), 85-112, Univ. Liege Press. 
Jewitt, D. C. and Luu, J. X. : 1992, "Submillimeter continuum observations of 2060 Chiron." Astron. J., 104, 398-404.

Jewitt, D. C. and Luu, J. X. : 1993a, "The discovery of the slow moving object 1992 QB 1 ." Nature, 362, 730-732.

Jewitt, D. C. and Luu, J. X. : 1993b, "IAU Circular 5865."

Johnson, R. E., Cooper, J. F., Lanzerotti, L. J. and Strazzulla, G. : 1987, "Radiation formation of a non-volatile comet crust." Astron. Astrophys., 187, 889-892.

Joss, P. C. : 1973, "On the origin of short-period comets." Astron. Astrophys., 25, 271273.

Khare, B. N., Thompson, W. R., Murray, B. G. J. P. T., Chyba, C. F. and Sagan, C. : 1989, "Solid organic residues produced by irradiation of hydrocarbon-containing $\mathrm{H}_{2} \mathrm{O}$ and $\mathrm{H}_{2} \mathrm{O} / \mathrm{NH}_{3}$ ices : infrared spectroscopy and astronomical implications." Icarus, 79, 350.

Kowal, C. : 1989, "A solar system survey." Icarus, 77, 118.

Kuiper, G. P. : 1951, "On the origin of the solar system." In Astrophysics (J. A. Hynek, Ed.), 357-427, McGraw-Hill, New York.

Levison, H. F. : 1991, "The long-term dynamical behavior of small bodies in the Kuiper belt." Astron. J., 102, 787-794.

Levison, H. and Duncan, M. : 1990, "A search for proto-comets in the outer regions of the solar system." Astron. J., 100, 1669-1675.

Levison, H. and Duncan, M. : 1993, "The gravitational sculpting of the Kuiper belt." Astrophys. J. Lett., 406, L35-L38.

Luu, J. X. : 1993, "Spectral diversity in cometary nuclei." Icarus, 104, 138-148.

Luu, J. X. and Jewitt, D. C. : 1988, "A two-part search for slow moving objects." Astron. J., $95,1256$.

Luu, J. X. and Jewitt, D. C. : 1990, "Cometary activity in 2060 Chiron." Astron. J., 100, 913-923.

Luu, J. X. and Jewitt, D. C. : 1993a, "IAU Circular 5730."

Luu, J. X. and Jewitt, D. C. : 1993b, "IAU Circular 5867."

Marsden, B. : 1993, "Minor Planet Circular 22594."

Meech, K. and Belton, M. : 1989, "IAU Circular 4770."

Meyer, P., Ramaty, R. and Webber, W. R. : 1974, Cosmic ray astronomy with energetic particles." Physics Today, 27, 23-32.

Moore, M. H., Donn, B., Khanna, R. and A'Hearn, M. F. : 1983, "Studies of protonirradiated cometary-type ice mixtures." Icarus, 54, 388-405.

Mueller, B. E. A., Tholen, D. J., Hartmann, W. K. and Cruikshank, D.P. : 1992, "Extraordinary colors of asteroidal object 1992 AD." Icarus, 97, 150-154.

Oikawa, S. and Everhart, E. : 1979, "Past and future orbit of 1977 UB, object Chiron." Astron. J., 84, 134-139.

Oort, J. H. : 1950, "The structure of the cloud of comets surrounding the solar system and a hypothesis concerning its origin." Bull. Astr. Inst. Neth., 11, 91.

Safronov, V. S. : 1972, "Ejection of bodies from the solar system in the course of the accumulation of the giant planets and the formation of the cometary cloud." In The Motion, Evolution of Orbits and Origin of Comets (G. A. Chebotarev, E. I. Kazimir, B. G. Marsden, Eds.), 329-334, Reidel, Dordrecht.

Stern, S. A. and Shull, J. M. : 1988, "The thermal evolution of comets in the Oort cloud by stars and supernovae." Nature, 332, 407-411.

Strazzulla, G., Baratta, G. A., Johnson, R. E. and Donn, B. : 1991, "Primordial comet mantle : irradiation production of a stable, organic crust." Icarus, 91, 101-104.

Strazzulla, G. and Johnson, R. E. : 1991, "Irradiation effects on comets and cometary debris." In Comets in the Post-Halley Era (R. L. Newburn, Jr., M. Neugebauer, J. Rahe, Eds.), 243-276, Kluwer, Dordrecht.

Suess, S. T. : 1990, "The heliopause." Reviews of Geophysics, 28, 97-115.

Tholen, D. J., Hartmann, W. K. and Cruikshank, D. P. : 1988, "IAU Circular 4554." 
Tombaugh, C. W. : 1961, In Planets and Satellites (G. P. Kuiper, B. M. Middlehurst, Eds.), 12-30, University of Chicago, Chicago.

Torbett, M. V. and Smoluchowski, R. : 1990, "Chaotic motion in a primordial comet disk beyond Neptune and comet influx." Nature, 345, 49-51.

Webber, W. R. : 1987, "The interstellar cosmic ray spectrum and energy density. Interplanetary cosmic ray gradients and a new estimate of the boundary of the heliosphere." Astron. Astrophys., 179, 277-284.

Whipple, F. L. : 1964, "The history of the solar system." Proc. Natl. Acad. Sci. USA, 51 , 711-718.

Whipple, F. L. : 1977, "The constitution of cometary nuclei." In Comets, Asteroids, Meteorites (A. H. Delsemme, Ed.), 25-35, University of Toledo Press, Toledo.

Williams, I., Fitzsimmons, A., and O'Ceallaigh, D. : 1993, "IAU Circular 5869."

Wilson, P. D., Sagan, C., and Thompson, W. R. : 1994, "The organic surface of 5145 Pholus : constraints set by scattering theory." Preprint.

Wisdom, J. : 1985, "Meteorites may follow a chaotic route to Earth. Nature, 315, 731-733.

Yabushita, S. : 1993, "Thermal evolution of cometary nuclei by radioactive heating and possible formation of organic chemicals." Mon. Not. Roy. Astron. Soc., 260, 819-825.

Yeomans, D. K. : 1986, In Proc. 20th ESLAB Symp., p. 419, ESA SP-250, Heidelberg. 